seen at age 2 months because of hypotonia, delayed motor development and lactic acidosis. Decreased activities of both cytochrome c oxidase and long chain acyl coenzyme A dehydrogenase were found in a muscle specimen of 11 months and in a liver specimen obtained post mortem at 13 months. Myoclonic seizures accompanied by multifocal EEG epileptic discharges were treated with valproate. Seizures were controlled, but her general condition deteriorated within 2 months, respiratory insufficiency worsened and the acidosis became intractable. Post mortem examinations showed severe demyelination in the capsula interna, calcification in the brain stem ganglia, and gliosis in the pons and medulla. The liver was enlarged with centrolobular fatty infiltration. A rare occurrence of two independent mitochondrial enzyme defects is suggested (Reichmann H et al. Cytochrome c oxidase deficiency and long-chain acyl coenzyme A dehydrogenase deficiency with Leigh's subacute necrotizing encephalomyelopathy. Ann Neurol Jan 1992; 31:107-109). (Correspondence: Dr. Reichmann, Department of Neurology, University of Würzburg, Josef-Schneider-Strasse 11, D-8700 Würzburg, Germany.)

COMMENT. Valproate administration was not considered the cause but may have worsened the palmitoyl CoA dehydrogenase deficiency. Leigh's syndrome, first described in 1951, appears to be non-specific biochemically as well as clinically. Consistent early clinical features in the infantile cases are a quiet immobility with lack of crying and hypotonia. In addition to the COX deficiency and dehydrogenase deficiency described above, defects of the pyruvate dehydrogenase multi-enzyme complex and pyruvate carboxylase have been reported.

\title{
FAMILIAL SPASTIC PARAPARESIS AS A MITOCHONDRIAL DISORDER
}

A girl presenting at 6 years with familial spastic paraparesis and having deficiencies of respiratory chain enzyme complex I, III and IV is reported from the Departments of Pediatrics and Neurology, Loyola University Medical Center, Maywood, IL. Her gait was clumsy and her maternal grandmother had a spastic gait with onset at age 40 . Deep tendon reflexes were brisk at the knees. Plantar responses were extensor bilaterally and ankle clonus was unsustained. The tone was increased in both lower extremities. The gait was wide based with hip circumduction. A 3 centimeter cyst in the left parietal region on MRI appeared to be coincidental. Efforts to treat the deficiencies of mitochondrial enzymes with various respiratory chain co-factors (coenzyme $\mathrm{Q}$, vitamin $\mathrm{K}$ and $\mathrm{C}$ ) and a mitochondrial substrate entry carrier (carnitine) were rewarded with no further progression of symptoms and some mild improvement clinically. The mitochondria were structurally normal by electron microscopy and ragged red fibers were absent on muscle biopsy (Beltran RS, Coker SB. Familial spastic paraparesis: a case of a mitochondrial disorder. Pediatr Neurosurg Oct 1991; 16:40-42). (Reprints: Dr. Beltran, Department of Pediatrics, Loyola University Medical Center, 2160 South First Ave., Maywood, IL 60153.)

COMMENT. Familial spastic paraparesis or Stümpell's disease is characterized by a progressive gait disturbance with lower extremity 
spasticity and weakness, without associated sensory, cerebellar or cranial nerve deficits. The age of onset ranges from 1-55 years and varies within specific kindreds. Heredity may be autosomal dominant, autosomal recessive or sex-linked. Involvement of the spinal cord is unique for a mitochondrial disorder.

\section{SEIZURE DISORDERS}

\section{EPILEPSY IN A MITOCHONDRIAL DISORDER}

Epilepsy in $9(22 \%)$ of 37 members of a family with maternally inherited mitochondrial disease is reported from the Department of Neurology, University Hospital, Tromsö, Norway. The epilepsy began in infancy or childhood in 3 patients and during adult life in the remainder. The different clinical expressions of epilepsy in the family were myoclonus, partial epilepsy and generalized tonic-clonic seizures. The neuropsychiatric disorders included intellectual deterioration, muscle weakness, pigmental retinal degeneration, progressive hearing loss, cerebellar ataxia and in 1 patient a hemiplegia and paranoid psychosis. The CT scan showed cerebral atrophy, cerebellar atrophy and calcification in the basal ganglia. The EEG abnormalities included slowing and spikes, sometimes focal, and a bilateral paroxysmal response to photic stimulation. A muscle biopsy from the proband revealed a mild defect in the NADH-ubiquinone oxidoreductase step (complex 1 ) in the respiratory chain (Torbergsen $T$ et al. Epilepsy in a mitochondrial disorder. $\rfloor$ Neur Neurosurg \& Psych Dec 1991; 54:1073-1076). (Correspondence: Dr. Torbergsen, Department of Neurology, 9038 University Hospital, Tromsö, Norway.)

COMMENT. The various clinical manifestations of epilepsy including partial motor and/or complex partial epilepsy in different branches of this family were remarkable and worthy of publication. The authors postulate that mitochondrial dysfunctions may be the cause of some epileptic syndromes of undetermined etiology.

\section{CSF TRYPTOPHAN IN INFANTILE SPASMS}

The levels of tryptophan (TRP) metabolites in the CSF of 8 patients with infantile spasms are reported from the Department of Pediatrics, St. Marianna University School of Medicine, Kawasaki 213, Japan. Concentrations in patients were compared to TRP metabolites in CSF from 20 age matched controls (mean age: 5.8 months). The levels of CSF serotonin, 5-hydroxyindoleacetic acid and kynurenine were significantly lower in infantile spasm patients compared to controls, whereas the levels of CSF 3hydroxykynurenine was significantly higher in patients. Tryptophan and 5hydroxytryptophan were not significantly different in patients compared to controls. The CSF was collected before treatment. The findings suggest that infantile spasms are associated with a decrease in serotonergic metabolites (Yamamoto H, Studies on CSF tryptophan metabolism in infantile spasms. Pediatr Neurol Nov/Dec 1991; $\underline{7}: 411-414$ ). (Correspondence: Dr. Yamamoto, 